\title{
Intrapericardial haematic cyst A rare form of left superior vena cava atresia
}

\author{
A CABRERA, P MARTINEZ, A DEL CAMPO \\ From the Departments of Paediatrics, Paediatric Cardiology and Surgery, and Cardiovascular Surgery, Ciudad \\ Sanitaria Enrique Sotomayor, Cruces, Vizcaya, Spain
}

SUMMARY A 21 month old infant was found to have an intrapericardial haematic cyst at operation. This rare entity may be explained by a pouch-like dilatation of an atretic left superior vena cava.

Intrapericardial masses are rare in childhood, and most often consist of teratomas and bronchogenic cysts. ${ }^{1}$ The vast majority are associated with serosanguinous pericardial effusions and congestive heart failure from cardiac tamponade. ${ }^{23}$ We report an infant, initially diagnosed as having an intrapericardial teratoma by cross sectional echocardiography, ${ }^{4}$ in whom surgical exploration showed a pouch-like venous dilatation arising from an atretic left superior vena cava and connected to the left innominate vein. ${ }^{5} \mathrm{We}$ believe this case represents the first description of such anomaly.

\section{Case report}

A 21 month old female infant was admitted because of fever, cough, facial puffiness, respiratory distress, and congestive heart failure. She had been the product of a full-term pregnancy and normal delivery. Both parents were healthy. Two hours after birth she presented with hypotonia and respiratory distress. A cardiopulmonary arrest ensued, but she was successfully resuscitated. Clinical follow up was, however, uneventful until her present admission.

Her weight was $12 \mathrm{~kg}$, length $88 \mathrm{~cm}$, and head circumference $52 \mathrm{~cm}$. Physical examination showed a quiet precordium with muffled heart sounds, decreased breath sounds bilaterally, and an enlarged liver (palpable $3 \mathrm{~cm}$ below the costal margin). Femoral and axillary pulses were normal. A chest radiograph showed a very enlarged heart and a hazy left hemithorax (Fig. 1). The electrocardiogram showed decreased voltage in the precordial leads. $M$

Requests for reprints to Dr A Cabrera, Alameda Recalde 35B, Bilbao 11, Spain.

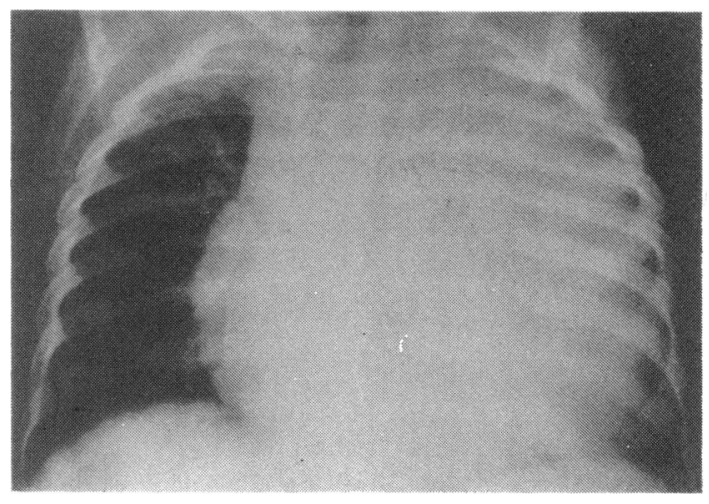

Fig. 1 Chest radiograph showing cardiomegaly, a hasy left hemithorax, and a pericardial effusion.

mode echocardiography suggested an anterior and posterior pericardial effusion, and a cross sectional echocardiogram showed a large pericardial effusion and a round cyst like echo $(4.45 \times 3.65 \mathrm{~cm}$ in diameter) on the left lateral border of the heart but independent of it (Fig. 2). Serum lactate dehydrogenase activity was $285 \mathrm{IU} / 1$ and serum creatine phosphokinase activity $85 \mathrm{IU} / \mathrm{l}$. A tuberculin (purified protein derivative) intradermal test and Weinberg's and Casoni's tests were all negative. Pericardiocentesis was performed and $80 \mathrm{ml}$ of a serosanguinous fluid obtained. Aerobic and anaerobic cultures, including specific cultures for mycobacteria, were negative. Cytological examination showed hemosiderin-loaded histiocytes.

The pericardium was approached surgically through a left thoracotomy. About $100 \mathrm{ml}$ of fluid was present in the pericardial sac. The egg shaped mass 


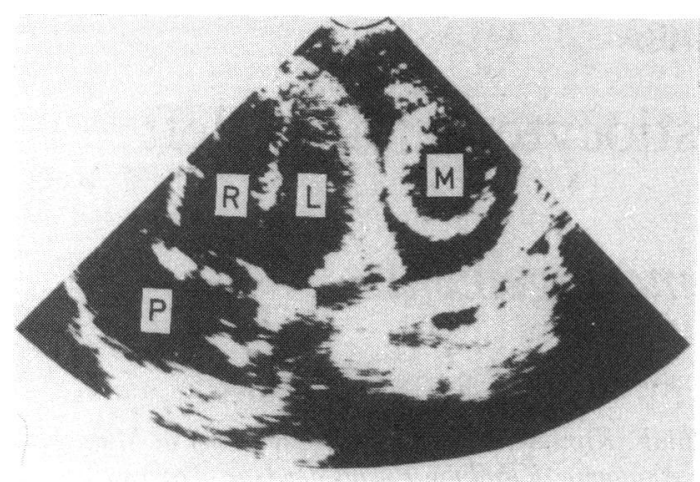

Fig. 2 Cross sectional echocardiogram in the apical four chamber view showing a pericardial effusion $(P)$, moderate atrial compression, and a cystic mass $(M)$ on the left of the heart but separated from it. $R$, right atrium; $L$, left atrium.

was $4.5 \times 3.5 \mathrm{~cm}$ in diameter and was connected by a patent venous pedicle to the left innominate vein. Ligation and resection of the cystic mass and the pedicle walls showed a venous structure. After an uneventful clinical course the infant was discharged on the eighth postoperative day, when norml heart size was evident on the chest radiograph.

\section{Discussion}

During the early stages of embryonic development, at about the fourth week of intrauterine life, the anterior and posterior cardinal veins join together to form the right and left common cardinal veins. Two weeks later the innominate vein joins both right and left anterior cardinal veins. By the second month of life the embryo has a well structured venous system. The right superior vena cava, with the azygos vein, originates from the right anterior cardinal vein, right common cardinal vein, and supracardinal vein. The left anterior cardinal vein is obliterated and results in Marshall's ligament, whereas the coronary sinus remains patent and drains into the left atrium. ${ }^{5}$ Partial patency of the left anterior cardinal vein may form a rudimentary pouch like venous structure, which may eventually become large and dilated. Persistent haemorrhagic effusions from that venous pouch could explain the formation of the serosanguinous pericardial fluid. ${ }^{2}$

The differential diagnosis in infancy should include teratomas and bronchogenic cysts. 6 Teratomas may compress the superior vena cava, aorta, or pulmonary artery and are frequently calcified. ${ }^{67}$ They produce dense, thick echoes on $M$ mode echocardiograms at the level of the aortic root. ${ }^{6}$ Cross sectional echocar- diography can determine the size and shape of the teratoma, 478 and the diagnosis is facilitated by the presence of a pneumopericardium after pericardiocentesis. ${ }^{49}$ Bronchogenic cysts are occasionally very difficult to diagnose preoperatively 10 and may only be so after pathological examination of the resected mass. In retrospect, we failed to establish an accurate preoperative diagnosis because we rarely perform innominate vein angiography. Such an examination would have delineated the left superior vena cava and its terminal venous dilatation. The pathological examination confirmed that the mass had a venous structure and that the fluid was indeed blood.

In summary, during embryonic development the left superior vena cava may not be obliterated completely and may give origin to an intrapericardial cystic mass, which may disturb cardiac haemodynamics. Diagnosis of this anomaly is very difficult with routine non-invasive procedures, but the prognosis is excellent after appropriate surgery.

\section{References}

1 Deenadayalu RP, Tuuri D, Dewall RA, Johnson GF. Intrapericardial teratoma and bronchogenic cyst. $\mathcal{F}$ Thorac Cardiovasc Surg 1974; 67: 945-52.

2 Reynolds JL, Donahue JK, Pearce CW. Intrapericardial teratoma: a cause of acute pericardial effusion in infancy. Pediatrics 1969; 43: 71-8.

3 Torres-Aybar FG, Basora G, López-Busquets RH, Rodriguez-Estapé HF. Intrapericardial teratoma. A cause of cardiac tamponade in infancy. Bol Assoc Med PR 1975; 67: 366-8.

4 Farooki ZQ, Hakimi M, Arciniegas E, Green EW. Echocardiographic features in a case of intrapericardial teratoma. $\mathcal{~ Y C U ~ 1 9 7 8 ; ~ 6 : ~ 1 0 8 - 1 0 . ~}$

5 Cabrera A, Sota L, Pastor E, Chouza M. Vena cava superior irquierda. Estudio de 46 casos. Implicaciones anatomicas, clínicas y quirúrgicas. Rev Esp Cardiol 1982; 35: $535-40$.

6 Arciniegas E, Hakimi M, Farooki ZQ, Green EW. Intrapericardial teratoma in infancy. $\mathcal{F}$ Thorac Cardiovasc Surg 1980; 79: 306-11.

7 De Geeter B, Kretz JG, Nisand I, Eisenmann B, Kieny $\mathbf{M}$, Kieny $\mathbf{R}$. Intrapericardial teratoma in a newborn infant. Use of fetal echocardiography. Ann Thorac Surg 1983; 35: 664-6.

8 Zerella JT, Halpe DC. Intrapericardial teratomaneonatal cardio-respiratory distress amenable to surgery. f Pediatr Surg 1980; 15: 961-3.

9 Pernot C, Frisch R, Mathieu P, Olive D, Vidailhet $M$. Les teratomes intrapericardiques du nourrison: a propos de 2 observations avec succès chirurgical. Arch Mal Coeur 1968; 61: 546-52.

10 Engle DE, Tresch DD, Boncheck LI, Foley D, Brooks HL. Misdiagnosis of pericardial cyst by echocardiography and computed tomography scanning. Arch Intern Med 1983; 143: 351-2. 